\title{
PROPERTIES OF THE OPTOKINETIC MOTOR FIBRES IN THE ROCK LOBSTER: \\ BUILD-UP, FLIPBACK, AFTERDISCHARGE AND MEMORY, SHOWN BY THEIR FIRING PATTERNS
}

\author{
BY B. YORK,* C. A. G. WIERSMA AND K. YANAGISAWA $\dagger$ \\ Division of Biology, California Institute of Technology, \\ Pasadena, California, U.S.A. 91 109
}

(Received II fanuary 1972)

\section{INTRODUCTION}

Motor fibres which cause eye movement in the horizontal plane in response to optokinetic stimuli have been demonstrated in the crab, Carcinus (Burrows \& Horridge, 1968; Wiersma \& Fiore, 1971 $a, b$ ) and in the crayfish, Procambarus (Wiersma $\&$ Oberjat, 1968). Such optokinetic movements are limited to an angle of up to about I $5^{\circ}$. Periodically the eyes flick back toward their starting point and resume their slow motion. Motor fibres responsible for horizontal eye movements which have input from the statocysts, either solely or in combination with visual input, are known only in Carcinus (Wiersma \& Fiore, 1971 $b$ ). Here, one large phasic fibre shows only statocyst input in that it responds to rotation of the animal in the horizontal plane in one direction, and to the stopping of rotation in the other, in either light or darkness. A second fibre responds similarly to rotation in the dark, but with a lower threshold, as well as to moving objects travelling in the other direction over the eye's visual field, whereas the third responds solely to visual input. In the crayfish rotation in the dark is without influence on any of its optokinetic fibres.

This study on analogous motor fibres in the rock lobster shows that, as in the crayfish, these units have no statocyst input. However, in addition to visual influences, antennal movement has a weak effect on the fibres. The possible significance of the latter input in the antennal 'pointing' reactions of the lobster will be discussed.

In addition, neurophysiological events in these units will be described which appear to underlie an 'optokinetic memory' similar to that reported in the crab, as shown by eye-position measurements, by Horridge $(1966 a, b ; 1968)$. The presence of such a memory is demonstrated in the lobster by the fact that about the same firing rate caused by standing contrasting stripes in the visual field was resumed after a short period of darkness and, even more compelling, adjusted to the new situation when the stripes had been moved clockwise or anticlockwise by a few degrees. From the behaviour of the lobster motor neurones it is obvious that the strength of this memory is very variable from animal to animal.

- Present address: Department of Physiology and Biochemistry, University of Southampton, Southampton, England.

$\dagger$ Present address: Department of Physiology, Tsurumi Women's University, Yokohama, Japan. 


\section{MATERIALS AND METHODS}

Animals used were Californian rock lobsters Panulirus interruptus (Randall). They were maintained in a seawater aquarium at $14{ }^{\circ} \mathrm{C}$ and were exposed to a $\mathrm{I} 2 \mathrm{~h}$ light/dark cycle (7 a.m.-7 p.m.).

For experimentation, the animal was suspended in air by a clamp which was applied to the posterior carapace, after the abdomen had been wrapped in a wet cloth. The antennae were usually held backwards with rubber bands, but were freed for some experiments. An animal was suspended in a metal drum of 19 -inch diameter for most experiments, with the midpoint between the two eyes coinciding as nearly as possible with the axis of rotation. The interior of the drum was light grey, but this was usually covered with a card of black and white stripes, subtending an angle of $25^{\circ}$ at the eye. Black and white targets of varying sizes could be fixed on the inside of the drum by means of an array of magnets on the outside. Either the animal or the drum could be rotated over a wide range of speeds. An even illumination in the drum could be adjusted continuously up to about 20 foot-candles. In some experiments a hand-held light source $\mathrm{r} \mathrm{mm}$ in diameter was used.

Single-unit recording was carried out by inserting an electrolytically sharpened, insulated, steel insect pin (oo) into the oculomotor nerve by hand. A total of four approaches were used for each animal on separate days, namely a low and a peripheral lead for each side, as previously described (Wiersma \& Yanagisawa, 1971). Each experimental session lasted for about $3 \mathrm{~h}$. Recording techniques were the standard ones used in this laboratory (e.g. Wiersma \& York, 1972).

\section{RESULTS}

As in other stalk-eyed decapods two sets of optokinetic fibres are present. For each set, up to four fibres, differing in firing rates and thresholds, could be recorded in one lead. Usually the largest signal in any lead is from the most phasic fibre which, in general, shows the highest threshold. Under resting conditions, low-level activity is usually present in the more tonic fibres. One set is excited by anticlockwise motion (ACM) of the animal in the horizontal plane, and inhibited by clockwise motion, whereas the other is excited by clockwise motion (CM) and inhibited by anticlockwise motion. The influence of visual stimuli on both sets is modulated to a considerable extent by the excited state and to a lesser extent by antennal, and perhaps antennule, joint input. For instance, moving both antennae clockwise caused weak excitation of the ACM fibre, and thus resulted in an eye movement in the clockwise direction, whereas anticlockwise movements of the antennae inhibited the ACM fibre (Fig. I). Habituation to antennal input occurs slowly.

We never saw any indication of the presence of peripheral inhibitory fibres or of joint receptors at the level of the outer eyecup, in agreement with the findings in crayfish and crabs. Rapid rotation of the animal in the dark fails to affect the firing rate of any of the optokinetic fibres. Thus, in contrast to Carcinus (Wiersma \& Fiore, 197 1 ), but as in the crayfish (Wiersma \& Oberjat, 1968), there is no statocyst input onto these fibres.

For visual inputs, we found no significant differences with regard to the reactivity 


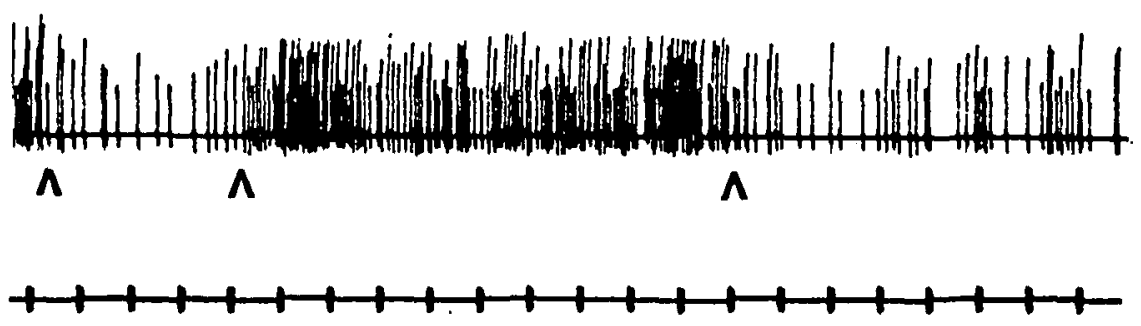

Fig. I. Antennal joint input onto a tonic anticlockwise fibre. Ist and 3 rd arrow: anticlockwise rotation of both antennae. 2 nd arrow: clockwise rotation of both antennae. Time base: 1 sec.

Table 1. Contralateral visual input onto clockwise and anticlockwise fibres

$\begin{array}{ccc}\text { No input } & \text { Weak input Strong input } \\ 24 \text { total } & 19 & 9\end{array}$

- These fibres had known optic fibres in the same lead.

of comparable fibres. Thus, though the clockwise motor fibres of the two eyes innervate functionally different muscles, their thresholds and firing rates were not noticeably different for the same input circumstances.

The relative effectiveness of optokinetic visual input from the ipsilateral and contralateral eyes varies greatly between preparations (Table $\mathbf{I}$ ). This is true for reactivity to small moving light sources as well as for rotating stripes. In many cases the contralateral input is either absent or very weak, but occasionally it approaches or even surpasses that of the ipsilateral eye. Although the total surface of either eye potentially provides for input, in some cases marked differences in reactivity were noted. This was most easily demonstrated with a small moving light, which could cause more impulses when travelling, e.g. over the dorsal than the ventral part of the eye, whereas in other cases the front half of the eye was more effective than the back. As in the crayfish, the effects of the two eyes are additive, as can be shown by the fact that a single stripe elicits the strongest response when it is within the visual fields of both eyes. Also, the frequency evoked by a stripe pattern with both eyes uncovered was approximately the sum of those obtained from each eye separately.

The intensity of the reaction to vertical edges moving across the eye appears to be determined mainly by their summed length. For instance, black targets, $15^{\circ}$ in visual angle, scattered randomly over the inner surface of the drum, gave rise to approximately the same response frequency at equal drum speeds as when they were arranged to form vertical $15^{\circ}$ stripes. Edges moving exactly in the vertical axis of the eye have neither an excitatory nor an inhibitory influence, but as soon as a horizontal vector of movement is present, the discharge rate will be affected.

When a striped drum is turned in the preferred direction after having been stationary, there is a gradual increase in firing frequency in the tonic optokinetic fibres. This increase was not always smooth, and in about $50 \%$ of the preparations it took the form of a definite bursting discharge which became masked when higher frequencies were reached but reappeared as the frequency declined on reversing the 


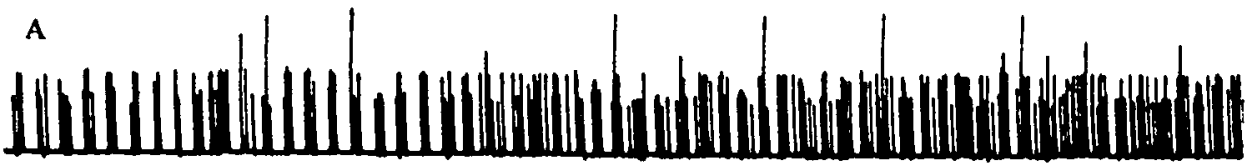
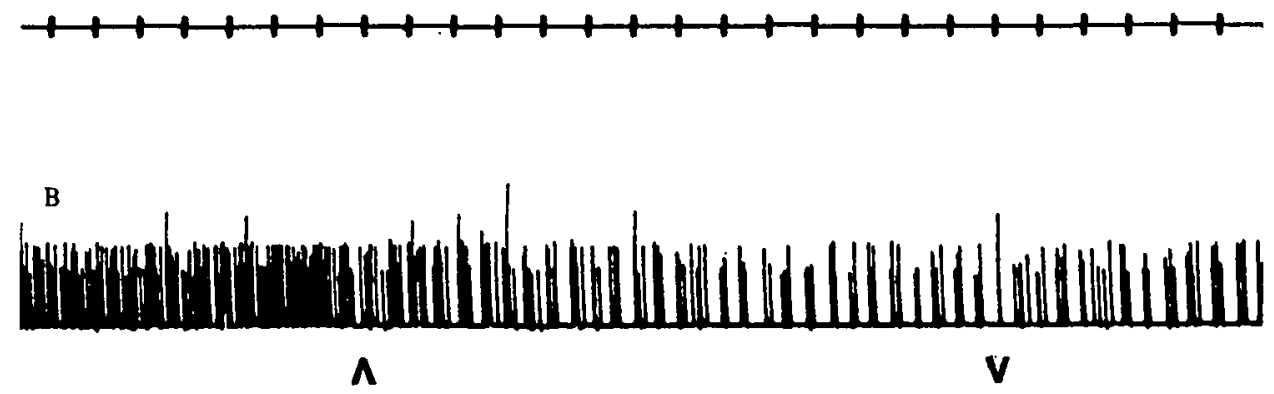

Fig. 2. Bursting discharge of a tonic anticlockwise fibre in response to rotating stripes and after rotation: (A) stripe rotation in the preferred direction, (B) stripe rotation in the unpreferred beginning at ist arrow and ending at and arrow. Time base: $\mathrm{I}$ sec.

Table 2. Afterdischarge in an anticlockwise motor fibre

$\begin{array}{cc}\begin{array}{c}\text { Time after } \\ \text { stopping } \\ \text { drum } \\ \text { (min) }\end{array} & \begin{array}{c}\text { Frequency } \\ \text { (impulses/ } \\ \text { sec) }\end{array} \\ 0-5 & 60-50 \\ 10 & 45 \\ 15 & 40 \\ 20 & 38 \\ 25 & 36 \\ 30 & 33 \\ 35 & 31 \\ 40 & 30\end{array}$

drum. The frequency of this bursting was not constant for any preparation but often speeded up from about $2 / \mathrm{sec}$ to $5 / \mathrm{sec}$ as the drum turned in the positive direction, and slowed down as the drum was reversed. Such bursting could also continue in the stationary striped drum (Fig. 2). In a few preparations, the bursts were synchronized with small but visible eye movements in the horizontal plane whereas in others no such eye motions could be detected.

\section{Optokinetic memory}

Once a high frequency has been built up in the optokinetic motor fibres by rotation in the preferred direction, stopping the movement will lead to an after-discharge whose duration is quite variable between preparations, lasting from only a few seconds to well over an hour (Table 2). If the motion is stopped when the firing frequency is low, due to a just-preceding flipback or to inhibition caused by rotation in the unpreferred direction, the discharge stays below the background frequency for some time. 


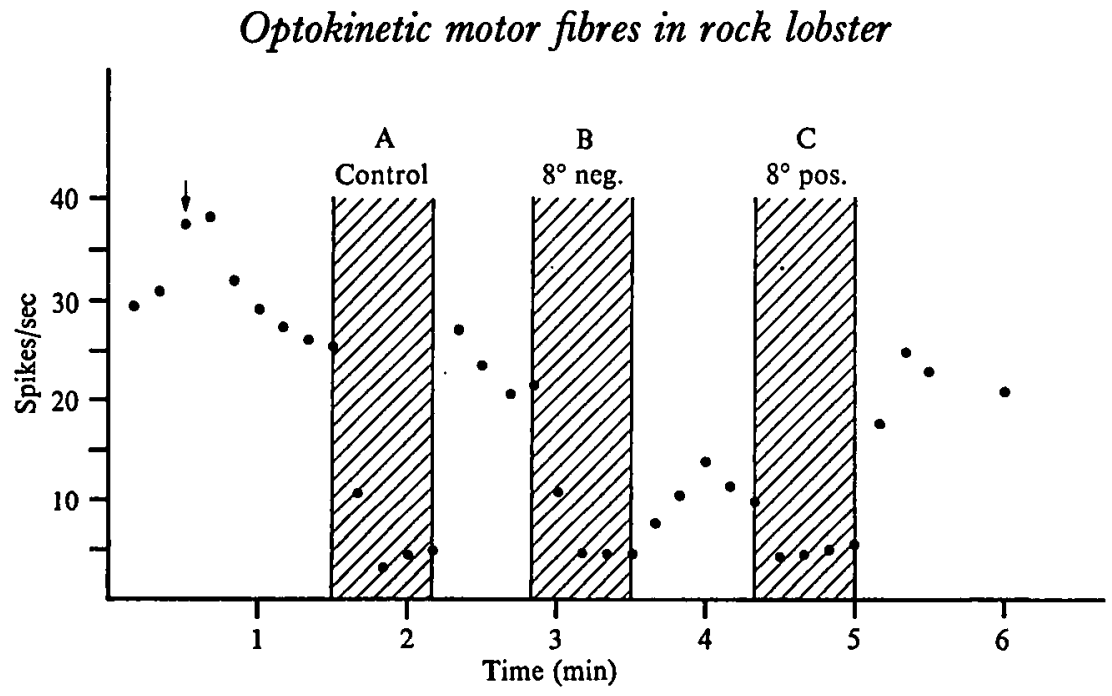

Fig. 3. Changes in the discharge frequency of a tonic clockwise fibre in a stationary drum caused by movement of stripes during $40 \mathrm{sec}$ darkness (hatched areas). Arrow denotes stopping stripe rotation after movement in the preferred direction. Stripe width was $25^{\circ}$. Drum movement during darkness: (A) No movement. After 'light on' firing rate is resumed at about the original level. (B) $8^{\circ}$ unpreferred direction, i.e. the leading edges of the stripes have been moved $8^{\circ}$ clockwise. After 'light on' an increase occurs, but to a level well below the original one. (C) $8^{\circ}$ preferred direction, i.e. the leading edges of the stripes have been moved $8^{\circ}$ anticlockwise. After 'light on' the frequency now increases to about control level again.

When the light is turned off during the positive afterdischarge, the firing rate usually assumes a level near to that of background or below. The rate of change can be very rapid or fairly gradual. On re-illumination there is a gradual resumption of a new firing rate over a period of $15-30$ seconds which is often equal or near to that before darkness (Fig. 3 A).

A real 'memory' of the stripe position relative to the eye rather than the eye position itself seems to be involved in this resumption of firing rate. This is indicated by the fact that the renewed firing rate is influenced by changing the position of the striped drum during darkness. When the drum was turned a few degrees in the unpreferred direction the firing rate reached about $15 \mathrm{sec}$ after re-illumination was significantly below that prevailing before the onset of darkness (Fig. ${ }_{3} \mathrm{~B}$ ). On the other hand, when the stripes were moved in the preferred direction the new firing level was higher than previously (Fig. $3 \mathrm{C}$ ). The period of darkness over which a 'memory' of stripe position could be maintained was determined by noting when obvious changes in firing frequency in response to stripe movement in the dark were still present. This time was found to be in the order of $2 \mathrm{~min}$. Such a memory system was shown only in $30 \%$ of the animals investigated, and was associated with the ability of the fibres to maintain a prolonged increase or decrease above background frequency after stopping the rotation of the striped drum under constant illumination.

\section{Influence of speed of movement}

The speed at which stripes are turned around the animal, or the animal is rotated in a stationary drum, has a great influence on the discharge pattern of the optokinetic motor fibres and the frequency of flipbacks. The fibres show habituation to the con- 


\section{Table 3. Influence of drum speed on the maximum firing frequency of a tonic optokinetic fibre and on fipback frequency}

$\begin{array}{ccc}\begin{array}{c}\text { Drum speed } \\ (\% / \mathrm{sec})\end{array} & \begin{array}{c}\text { Maximum } \\ \text { tonic firing } \\ \text { frequency } \\ \text { (spikes/sec) }\end{array} & \begin{array}{c}\text { Flipbacks/ } \\ \text { 10 sec }\end{array} \\ 0.06 & 35 & 0 \\ 0.7 & 50 & 1.5 \\ 2.0 & 41 & 1.5 \\ 10.0 & 20 * & 0\end{array}$

* Maximum frequency after initial early burst.

tinued motion of a striped drum and to repeated drum movements, especially at speeds above $2 \%$ sec. Habituation is not usually very pronounced but can be large, especially when the drum is turned rather than the animal. Once habituation has been established longer-lasting reactions to continued drum movements can be obtained only at intermediate speeds of about $2 \% \mathrm{sec}$, whereas at both slower and faster speeds there is no sustained difference above background. For slow speeds the impulse frequency changes so little that the difference is not significant, while for faster speeds the frequency changes only at the start of the rotation and soon returns to near the background level.

When habituation is not pronounced, even very slow speeds of $0.06 \% \mathrm{sec}$ of stripe rotation in the preferred direction lead to a gradually increasing discharge frequency, first of the tonic and later of the more phasic fibres. It then takes $5 \mathrm{~min}$ or more before the maximum for a tonic fibre is reached. On the contrary, with fast drum speeds of $10 \% \mathrm{sec}$ or more, the maximum is reached in less than a second. For the tonic fibre the maximum was fairly constant for different drum speeds, but was greatest for intermediate speeds of about $1 \% \mathrm{sec}$. The response to very fast speeds of $10 \% \mathrm{sec}$ or above invariably was less than to slower speeds after an initial higher-frequency burst at the start of rotation (Table 3 ).

When the drum is rotated in the unpreferred direction immediately after rotation in the preferred direction, the decline in firing frequency of all the fibres is always considerably faster than the build-up, by a factor of 2 or more. Very rapid declines occur in those preparations in which the afterdischarge on stopping the drum is of short duration.

Flipback movements of the eyes are accompanied by characteristic firing patterns in the optokinetic fibres. When a flipback occurred during rotation in the preferred direction, there was a sudden drop in firing frequency followed by a build-up, first in the more tonic and then in the more phasic fibres (Fig. 4), at a speed proportional to, but faster than, the original build-up. During flipbacks due to stripe rotation in the unpreferred direction, bursts appear usually in both fibre types, but occasionally only in the tonic. These bursts usually last for $0.5 \mathrm{sec}$ or less, but may have a duration of up to $2 \mathrm{sec}$ in tonic fibres. The magnitude of the eye movement and the changes in frequency of the phasic and tonic fibres vary greatly; for drum rotation in the unpreferred direction the eye motion is roughly proportional to the size and duration of the burst. Rarely does complete inhibition, especially of tonic fibres, occur during rota- 

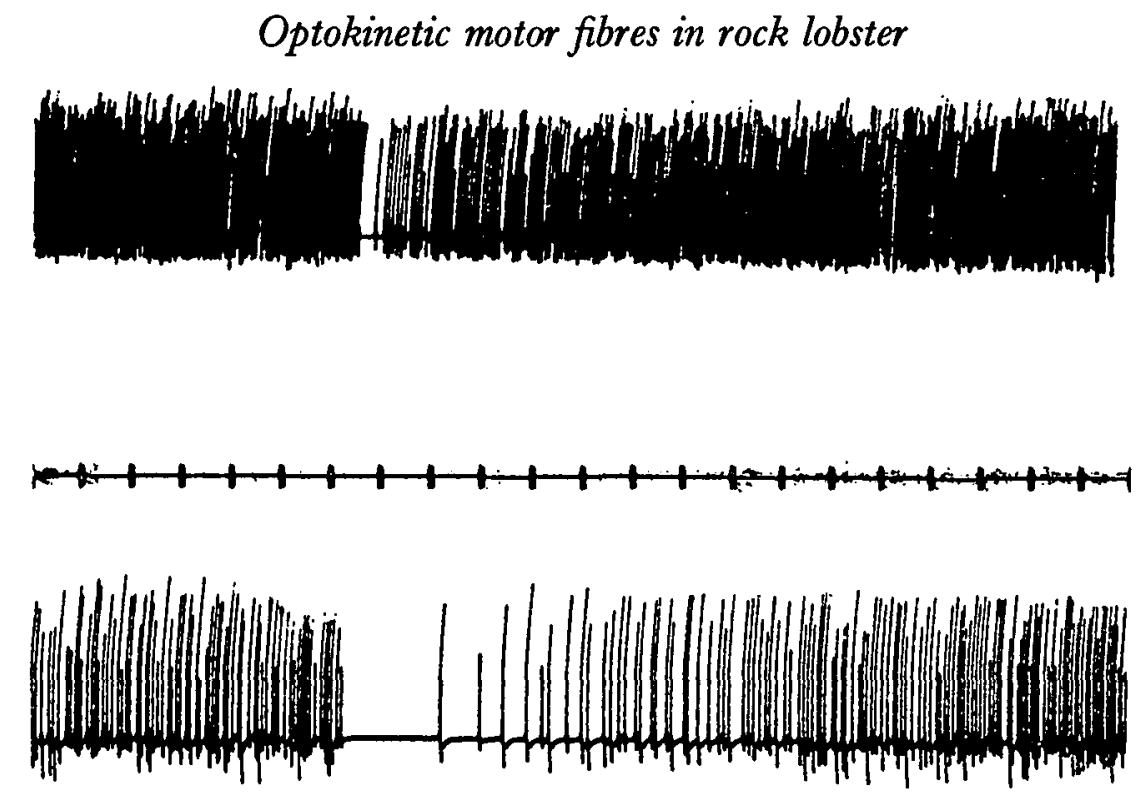

Fig. 4. Changes in firing frequency of a phasic and tonic clockwise fibre (recorded simultaneously) caused by a flipback during drum rotation in the preferred (anticlockwise) direction. These records were obtained by selecting for spike heights in the original tape. Drum speed: $0.06 \% \mathrm{sec}$. Upper trace: Tonic and phasic fibre discharges combined. Lower trace: Phasic fibre alone. Time base: I sec.

tion in the unpreferred direction, but since even isolated phasic spikes do not trigger observable eye movements, such low-frequency firing is likely of little functional significance.

The appearance of flipbacks is correlated with the attainment of a certain frequency level in the optokinetic fibres. In fresh preparations they are most frequent at fairly high drum speeds, but as habituation develops the speed range for the development of flipbacks becomes more restricted. Low drum speeds are then too slow to elicit the required frequency and at drum speeds above about $10 \% \mathrm{sec}$ habituation is too great (see Table 3). A flipback can be triggered in such instances, however, by a temporary increase resulting from an excited state. The decrease in firing frequency due to capping one eye, in preparations with bilateral input, can reduce or even prevent flipbacks. However, it is certain that the firing frequency is not the only factor that determines whether or not a flipback will occur.

The effectiveness of rotating the animal or the drum for stimulation of the optokinetic fibres varies considerably between animals. In roughly half the cases tested there was no appreciable difference, whereas in the rest, rotating the animal was noticeably more effective than rotating the drum. In some instances the difference was so great that drum rotation had no, or little, effect whereas animal rotation caused a strong discharge. This may be partly explained by the fact that when the animal is, for example, rotated in the preferred direction, all objects in the visual field move in the preferred direction relative to the eye, but when the drum is rotated in the preferred direction, objects in the upper $45^{\circ}$ of the visual field (above the drum) will cause inhibition because of the eye movement induced by the moving stripes. As previously mentioned, the fibres become habituated to rotation of the drum much faster 


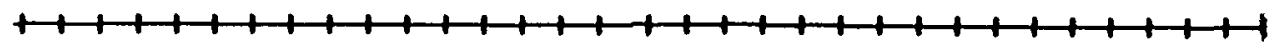

A

B

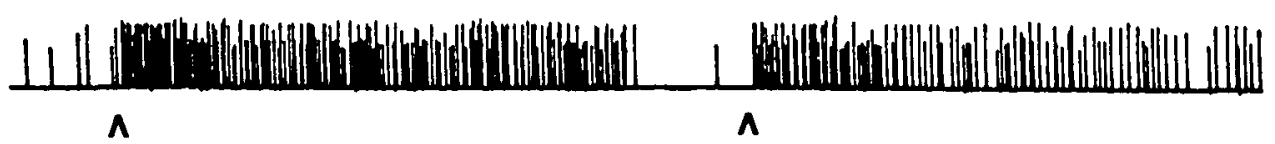

$\wedge$

$\wedge$
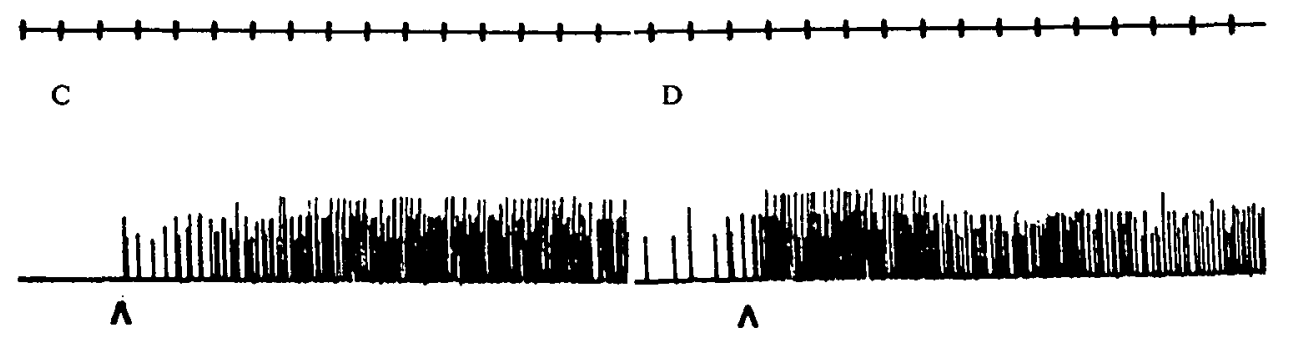

Fig. 5. Habituation of a tonic clockwise fibre to repeated drum rotation and dishabituation to drum rotation after animal rotation. (A) Response to drum rotation in the preferred direction. (B) Habituated response to repeated drum rotation. (C) Good response to animal rotation in the preferred direction. (D) Dishabituated response to drum rotation. Time base: I sec. Time interval between successive experiments was approximately $30 \mathrm{sec}$. Arrows denote onset of drum rotation.

than to rotation of the animal. Rotation of the animal even immediately after strong habituation to rotation of the drum is always effective and sometimes can dishabituate the response to drum rotation performed immediately afterward (Fig. 5).

\section{Optokinetically driven interneurones in the optic nerve}

The manner in which the motor fibres are driven by the optical input is still problematic in all species. In the rock lobster, though about nine classes of optically reacting interneurones are known to exist (Wiersma \& Yanagisawa, 1971), none of them by itself or in combination could provide the necessary input for the motor fibres. We have, however, found in the optic nerve itself signals of neurones which might provide for this purpose; but it is still uncertain whether these fibres are truly interneurones or branches of the tonic optokinetic motor fibres. There are several reasons why the first alternative seems likely. First, in all cases where it was certain that the fibre ran in the optic nerve, its input was always restricted to the ipsilateral eye, whereas for proven motor fibres this was the case in only $30 \%$. Secondly, these fibres did not respond when the capped ipsilateral eye clearly showed optokinetic reactions to contralateral input; thus they did not participate in driving the eye. In addition, these fibres were never accompanied by phasically responding fibres though this is often the case for tonic motor fibres.

They show the following properties, none of which distinguishes them clearly from motor fibres. Their discharge frequency is high when stimulated by movement, in 
omparison to most motor fibres. Like motor fibres, they are spontaneously active and are inhibited by movements in the unpreferred direction. It is of special interest that they do possess the capacity of after-discharge with a slow return to background frequency after rotation in the preferred and unpreferred directions. They respond well to moving light sources and to single targets down to $8^{\circ}$, and have even been found sensitive to single white targets occasionally.

\section{DISCUSSION}

It is always difficult, though especially so in the rock lobster, to judge the importance of optokinetic reflexes in the life of the animal. One obvious interpretation is that they serve more or less to stabilize the image on the eye when the animal makes a turning movement. However, according to Dijkgraaf (1956a), when such a movement is 'planned' it is accompanied by an initial eye movement in the turning direction, and would therefore make the whole optokinetic mechanism superfluous. The bilateral reflexes would then compensate only for passive dislocation of the animal by external agents, such as water currents. Although we have been unable to confirm his findings in the case of lobsters with chronically implanted electrodes, we used mainly visual observation only and did not measure eye angle. For a full discussion of the importance of this factor with regard to the orientation of the animal in space we refer to his papers (Dijkgraaf, $1956 a, b$ ).

The difficulties in understanding the role of the reflex do not end here, because even if normally present in voluntary turning, the fact that the two eyes are coupled to such varying degrees under different circumstances makes interpretation difficult. Indeed, there is often almost complete absence of any influence of contralateral input on the optokinetic motor neurones. However, in the absence of coupling it would become possible for the animal to track two separately moving objects, one before each eye, at the same time. That this in fact occurs is indicated by the open-field experiments on the antennal-pointing reactions by Lindberg (1955).

Since the optokinetic fibres were found to respond only to relatively large targets and to show considerable habituation to them, one may propose that they are not involved in tracking, especially of smaller objects, e.g. those below $8^{\circ}$ subtension. Note, however, that the visual acuity of the optical system as such is not limited to an $8^{\circ}$ angle since the 'seeing' fibres respond to moving targets of $4^{\circ}$ subtension or sometimes less, resulting in pointing of the antennae toward the target (Wiersma \& Yanagisawa, 197I). In contrast, the optokinetic fibres do not respond to stripes of this size moving at similar speeds. These two systems, that is the optokinetic tracking and the antennal reflex pointing, are therefore greatly independent of each other. Nevertheless, under certain circumstances they must mutually influence each other to some degree. For example, large single moving objects will cause both pointing and optokinetic reactions. The movement of the antenna during pointing will elicit impulses in the optokinetic fibre and orient the eye toward the object, so that the object will be seen longer by any given area of the eye's visual field. Such a feedback loop could lead to a 'focusing of attention' on the moving object. These influences are both weak and strongly habituating and thus likely to be of minor importance. However, they may offer one explanation why the 'seeing' fibres give a somewhat longer discharge to 
a large black target than to a smaller one moving at the same speed (Wiersma \& Yorl 1972).

Among the three or four optokinetic fibres present in one lead we have been unable to establish any differences in reactions other than that their thresholds are varied and related to their size. All can fire for a prolonged period during afterdischarge, though the larger, more phasic ones invariably stop sooner and start later for the same stimulus. Whether the tonic optokinetic fibre located in the optic nerve is really an input channel or whether it is a branch of one of the tonic motor fibres is uncertain. Most of its properties would be expected to be shown by a one-way conducting branch of a motor fibre.

The memory span of the motor fibres is much longer than that of the 'seeing' fibres under the same conditions. The discharge rate of the latter under constant illumination decreases relatively rapidly and returns to near baseline $30 \mathrm{sec}$ at most after initial presentation of a stationary target (Wiersma \& York, 1972). The motor fibres can remain firing at a high level to a stationary striped drum for $30 \mathrm{~min}$ or more after rotation stops. There is less difference, however, in their reactions concerned with 'forgetting' in the dark. 'Seeing' fibres can 'recognize' a changed situation, such as introduction of a target during darkness, providing the dark period does not exceed $30 \mathrm{sec}$; optokinetic fibres 'recognize' a change in stripe position providing the period of darkness does not exceed 2 min.

The fact that rotating the animal in a striped drum can sometimes have more effect on the frequency of the optokinetic fibres than rotating the drum, but at other times no difference is shown, suggests that an 'attention' factor may be present. The apparent visual stimulus is clearly different in the two cases, yet since objects above the rotating drum can often be 'ignored', such a factor must control the visual input to the fibres and 'focus attention' on only the stripes. The dishabituation of the optokinetic response to stripe movement following 'unexpected' animal rotation can also be considered as 'refocusing of attention' on the stripes.

The bursting that the optokinetic fibres show to a much greater extent than other optomotor fibres is of unknown origin. It is uncertain whether a feed-back loop with visual input is operative in the $50 \%$ of the preparations that clearly show bursting. In favour of this view is that tremor movements of the eye have been observed to accompany bursts, though this was not true in all cases. Horridge (1966c) has suggested that similar movements in crabs would modify the visual input, and be involved in the perception of edges and even perhaps in depth perception. However, these bursts cannot be essential since, for example, they did not occur in preparations which showed a pronounced memory. Another interpretation of such conspicuous bursts is to regard them as noise though this does not mean that small eye movements may never be necessary to prevent accommodation to visual input.

\section{SUMMARY}

I. The properties of sets of motor fibres responding to both clockwise and anticlockwise rotation have been studied in the oculomotor nerve of the rock lobster. There are probably three, but perhaps four, units in each set.

2. None of these fibres has statocyst input, but there is weak input onto the tonio 

chres from the antennal joints such that the eye turns in the direction toward which
the antenna points.

3. Many preparations show bilateral visual input onto all fibres but the degree of coupling between the eyes is very variable, and at times can be nearly totally absent.

4. Depending on the speed of rotation the fibres show a gradual build-up in frequency, during rotation in the preferred direction, interrupted by flipbacks. During the fast stage of the resulting nystagmic movements all agonistic fibres can be completely inhibited and all antagonistic ones can be activated, usually for a period of about $0.5 \mathrm{sec}$.

5. Fibre activity is demonstrated which appears to underlie an 'optokinetic memory' of contrasting target position in the visual field. It consists of $(a)$ very prolonged afterdischarges for a stationary striped pattern $(b)$ resumption of discharges at an appropriate frequency after dark periods up to $2 \mathrm{~min}$, and $(c)$ adjustment of such frequencies to changes in stripe position during the dark period.

6. The fibres show habituation to repeated stripe movement but the response can be dishabituated by passive rotation of the animal.

7. The largest visual responses were obtained to intermediate speeds of stripe rotation (about $2 \%$ sec).

This research has been supported by grants from the National Science Foundation (GB 693 I X) and the U.S. Public Health Service (NB 03627).

\section{REFERENCES}

Burrows, M. \& Horridge, G. A. (I968). Motoneurone discharges to the eyecup muscles of the crab Carcinus. F. exp. Biol. 49, $251-67$.

DijkGRAAF, S. (1956a). Kompensatorische Augenstieldrehungen und ihre Auslösung bei der Languste (Palinurus vulgaris). Z. vergl. Physiol. 38, 491-520.

DijKGraaf, S. (1956b). Ueber die kompensatorischen Augenstielbewegungen bei Brachyuren. Pubbl. Staz. Zool. Napoli $28,341-58$.

Horridge, G. A. (1966a). Optokinetic memory in the crab, Carcinus. F. exp. Biol. 44, 233-45.

Horridge, G. A. (I $966 b$ ). Optokinetic responses of the crab, Carcinus to a single moving light. 7 . exp. Biol. 44, 263-74.

HoRridGe, G. A. (1966c). Adaptation and other phenomena in the optokinetic response of the crab, Carcinus. J. exp. Biol. 44, 285-95.

HonRIDGe, G. A. (1968). Five types of memory in crab eye responses. In Physiological and Biochemical Aspects of Nervous Integration. Ed. F. D. Carlson, pp. 245-65. Englewood Cliffs: Prentice-Hall.

LindaerG, R. G. (1955). Growth, population dynamics, and field behavior in the spiny lobster, Panulirus interruptus (Randall). Calif. Univ. Publ. in Zool. 59, $157-247$.

Wiersma, C. A. G. \& FIORE, L. (1971 $a$ ). Factors regulating the discharge frequency in optomotor fibres of Carcinus maenas. F. exp. Biol. 54, 497-505.

WIERSMA, C. A. G. \& FIORE, L. (I $97 \mathrm{I} b$ ). Unidirectional rotation neurones in the optomotor system of the crab, Carcinus. F. exp. Biol. 54, 507-13.

Wiersma, C. A. G. \& Onerjat, T. (Ig68). The selective responsiveness of various crayfish oculomotor fibers to sensory stimuli. Comp. Biochem. Physiol. 26, 1-16.

Wiersma, C. A. G. \& Yanagisawa, K. (197I). On types of interneurons responding to visual stimulation present in the optic nerve of the rock lobster, Panulirus interruptus. $\mathcal{F}$. Neurobiol. 2, 292-309.

Wiersma, C. A. G. \& York, B. (1972). Properties of the seeing fibers in the rock lobster: Field structure habituation, attention and distraction. Vision Res. 12, 627-40. 
